\title{
Short hairpin RNAs artifactually impair cell growth and suppress clustered microRNA expression.
}

\author{
Powers $\mathrm{JT}^{1,2}$, da Rocha EL ${ }^{1,3}$, Pearson DS ${ }^{1,3}$, Missios $\mathrm{P}^{1}$, de Soysa TY1 , Barragan J1, \\ Cahan $\mathrm{P}^{4}$, and Daley $\mathrm{GQ}^{1-3, *}$
}

\begin{abstract}
1Division of Pediatric Hematology/Oncology, Boston Children's Hospital, Boston, Massachusetts 02115, USA. 2Department of Biological Chemistry \& Molecular Pharmacology, Harvard Medical School, Boston, Massachusetts 02115, USA. ${ }^{3}$ Harvard Stem Cell Institute, Cambridge, Massachusetts 02138, USA. ${ }^{4}$ Department of Biomedical Engineering, Johns Hopkins University School of Medicine, Baltimore, MD 21205. * to whom correspondence should be addressed: george_daley@hms.harvard.edu
\end{abstract}

Functional gene disruption is a central tenet of cancer research, where novel drug targets are often identified and validated through cell-growth based knockdown studies or screens. Short hairpin RNA (shRNA)-mediated mRNA knockdown is widely used in both academic and pharmaceutical settings. However, off-target effects of shRNAs as well as interference with endogenous small RNA processing have been reported ${ }^{1-3}$. We show here that lentiviral delivery of both gene-specific and non-targeting control shRNAs impair in vitro cell growth in a sequence independent manner. In addition, exogenous shRNAs induce a depressed cell-cycle-gene expression signature that is also shRNA-sequence independent and present across several studies. Further, we observe an shRNA mediated general repression of microRNAs belonging to polycistronic genetic clusters, including microRNAs from established oncogenic microRNA clusters. The collective impact of these observations is particularly relevant for cancer research, given the widespread historical use of shRNAs and the common goal of interrogating genes that regulate proliferation. We therefore recommend that when employing shRNA for target validation, care be taken to titrate shRNA dose, use hairpin-expressing controls, perform gene-of-interest rescue experiments and/or corroborate shRNA-derived results by small interfering RNA (siRNA) knockdown or CRISPR/Cas9-mediated genetic knockout. Minimizing these deleterious sequence independent effects will improve research fidelity and help address reported challenges in experimental reproducibility ${ }^{4}$.

Gene-targeting shRNAs are typically introduced into cell lines in vitro through lentiviral infection, leading to genomic integration and permanent expression of the hairpin construct. Functionally similar to siRNAs, which are directly loaded into the RISC complex, shRNA must first be transcribed and processed by cellular microRNA biogenesis machinery. ShRNAs are valued because they are easy to use and can affect long term knockdown of a gene, whereas siRNA knockdown is transient. CRISPR/Cas9-mediated knockout also results in a permanent genetic event, though in some cases knockdown studies are preferred to evaluate intermediate gene expression phenotypes and avoid severe phenotypes or lethality due to complete loss of gene function. While shRNA knockdown occupies an important niche between short term and permanent gene disruption, their use comes with caveats. In addition to off-target effects and impact on small RNA processing machinery, shRNA-induced liver and neuronal toxicity have been documented in vivo ${ }^{3,5}$. In addition, shRNA-mediated cytotoxicity has been implicated in MYCdriven hepatocellular carcinoma in mice ${ }^{6}$.

In our recent work on the role of $L I N 28 B$ and its microRNA target let-7 in neuroblastoma we described a reduced-growth phenotype produced by shRNA-mediated LIN28B knockdown that was not replicated by either siRNA or CRISPR/Cas9-mediated LIN28B knockout? ${ }^{7}$. To further explore this observed shRNA induced toxicity, we first compared the effects of shRNA- and siRNA-mediated LIN28B knockdown on the cell cycle profile of BE(2)C neuroblastoma cells (Fig. 
1a, b). We observed disturbed profiles in both non-targeting control and LIN28B-targeting shRNA infected cells (Fig. 1c). This effect was not observed in control or LIN28B-siRNA transfected cells, consistent with our previous work (Fig. 1d). MYCN-siRNA served a control for an altered cell cycle profile (Fig. 1d). We next tested this shRNA/siRNA relationship in K562 cells, a morphologically distinct leukemia cell line that also expresses LIN28B, and again observed that multiple LIN28Btargeting shRNAs reduced cell growth similar to the $A B L 1$ siRNA control, while two LIN28Btargeting siRNAs did not (Fig. 1e, f). We observed a similar pattern of shRNA, but not siRNA, induced growth impairment during the course of studies of the NAT10 acetyltransferase in BE(2)C cells (Supp. Fig. 1a, b). LIN28B has recently been implicated in pancreatic cancer, where pancreatic cell growth inhibition was demonstrated using LIN28B-specific shRNAs ${ }^{8}$. However, we observed that the same pancreatic cancer cell lines were entirely refractory to complete LIN28B protein loss mediated by CRISPR/Cas9 targeting of the LIN28B genetic locus with multiple sgRNAs (Supp. Fig. 2a, b). Together, these results suggest that exogenous shRNA expression may generally suppress cell growth independent of hairpin sequence, potentially leading to misleading experimental conclusions. Of note is the observation that the non-targeting control hairpin induced the same cell cycle defect as gene-targeting shRNAs (Fig. 1C).

To further understand the apparent sequence independent growth inhibition of shRNAs, we interrogated the effect of a panel of scrambled siRNA, GFP-targeting CRISPR/Cas9, and nontargeting shRNA controls on the growth of $\mathrm{BE}(2) \mathrm{C}$ and HeLa cells five days post treatment. This approach allowed us to test each platform for effects on proliferation that were independent of unintentional gene targeting side effects. We intentionally used a high concentration of siRNA (100nM), as well as an siRNA against RPL3, a target well documented to impair cell proliferation ${ }^{9}$ as a positive control for reduced cell growth. Multiplicity of infection (MOI) for lentiviral CRISPR/Cas9 and shRNA constructs ranged from low MOls of 1 to high MOls of 20. Neither scrambled sequence control siRNAs displayed growth abnormalities, similar to the mocktransfection, whereas the RPL3-targeting siRNA severely impaired cell growth in both cell types, as expected (Fig. 2a,b). Cas9 and Cas9/GFP-targeting-sgRNA infected cells responded similarly, with neither construct perturbing growth compared to mock-infection, regardless of $\mathrm{MOI}$. In contrast, scrambled sequence shRNA-expressing lentiviruses significantly reduced both $\mathrm{BE}(2) \mathrm{C}$ and HeLa cell proliferation over the 5 day experiment, in a dose-dependent manner at MOls of 10 and 20 (Fig. 2a, b).

We sought to gain insight into the observed sequence non-specific shRNA induced proliferative defect and performed global mRNA expression analysis on $\mathrm{BE}(2) \mathrm{C}$ cells infected with empty lentivirus, non-targeting control shRNA, or each of three LIN28B targeting shRNAs. Replicate samples showed high correlation within each condition (Fig. 3a). Given the proliferation phenotype driven by shRNAs, we first identified the cell cycle genes that were differentially regulated after shRNA transduction and noted that all four shRNA samples, again including the non-targeting control, displayed significant down regulation of cell cycle genes compared to empty vector (Fig. 3b). Furthermore, the most significantly effected Gene Ontology processes were almost universally cell cycle related in all four shRNA samples (Fig. 3c). These data are consistent with a model wherein high level exogenous shRNA expression has a sequence-independent effect on cell proliferation as demonstrated by consistent growth impairment and downregulation of cell cycle related processes (Fig. 2, Fig. 3c).

We compared our expression data to two existing shRNA-based microarray datasets; one from $B E(2) C$ neuroblastoma cells and the other from DU145 prostate cancer cells ${ }^{10,11}$. Correlative analysis within the two studies showed agreement within sample replicates (Supp. Fig. 3a, 4a). Differential gene expression analysis showed a preferential down-regulation of cell cycle genes, consistent with our own data (Supp. Fig. 3b, 4b). Gene Ontology analysis of the most significantly altered pathways also revealed a strong cell cycle signature in both datasets (Supp Fig, 3c, 4c). Indeed, Venn diagram overlap of downregulated Gene Ontology processes revealed 44 common 
to all the three datasets (Fig. 3d; Supp. Table 1). Of note, 42 of them were cell cycle processes. Moreover, the 18 most highly significant for each comparison were all cell cycle-related and showed strong agreement across the studies. Note the agreement between our control-shRNA pattern and those of the other two studies (Fig. 3e). These inter-study observations are consistent with our own data and support a model where small hairpin RNAs inhibit cell growth in a sequence-independent manner.

Following transcription, shRNAs are processed by the microRNA biogenesis machinery and have been reported to impact microRNA levels ${ }^{3,12,13}$. We therefore examined global microRNA expression in the same set of $B E(2) C$ shRNA transduced cells. Analysis of microRNA expression patterns revealed high correlation among all of the shRNA samples, including the nontargeting scrambled sequence control shRNA, which was more closely correlated with LIN28B shRNA samples than empty vector control. This pattern is consistent with observed sequenceindependent effects on proliferation (Fig. 1, Supp. Fig. 1, Fig.4a). Of the 50 most downregulated microRNAs in each sample set, 25 were consistent between control shRNA and all three LIN28B shRNA-expressing cells (Fig. 4b). Upon analysis of these 25 microRNAs, we discovered that 24 of them belonged to clustered microRNA loci (Fig. 4c). Moreover, when we examined expression of clustered vs. single-microRNA loci across the genome, we observed a strong global downregulation of clustered microRNA expression compared to singleton microRNAs (Fig. 4d). These data indicated that the broad cell growth impairment observed following transduction of high levels of shRNA correlated markedly with aberrant processing of clustered species of microRNA.

The microRNAs of the miR-17, miR-18, miR-19, and miR-25 families are highly expressed in multiple cancers. They are established pro-growth oncogenes, and are all transcribed from one of three OncomiR clusters ${ }^{14,15}$. Indeed, eight of these microRNAs are among the highest expressed microRNAs in BE(2)C cells (Supp. Fig. 5a). The miR-17-92 cluster is the best characterized of these and has been identified as a transcriptional target of $M Y C^{16}$. Upon shRNA infection of $\mathrm{BE}(2) \mathrm{C}$ cells, microRNA levels from the miR-17-92 OncomiR cluster were broadly suppressed in all shRNA samples, including non-targeting control shRNA (Fig. 4e). Further, we observed the same pattern of repression in the remaining two OncomiR clusters (Supp Fig. 5c, d). Given their importance in cancer biology, sequence-independent shRNA-induced suppression of these oncogenic microRNA clusters may offer a plausible explanation for the proliferative defects observed in our study.

Loss of gene function analysis is indispensable to cancer research. Within the Nature family of journals alone, chosen for analysis due to their high rate of depositing published manuscripts into pubmed central, there have been over three thousand publications in cancer research over the last ten years, well over a thousand of which have included gene knock-down data (Supp. Fig. 6a-c). Tool fidelity in such a widely used research approach is therefore critical. We demonstrate here that shRNA can impact cell growth in vitro as well as preferentially disrupt clustered microRNA expression in a sequence-independent manner. We further identify a depressed cell cycle Gene Ontology signature that is consistent across multiple shRNA studies. Our data poses a significant concern for use of shRNA knockdown for cancer research in particular, where the common goal is to identify genes whose knock-down compromises cell growth. This is particularly true for easy-to-infect cells, which include most adherent lines. Difficultto-infect cells, such as lymphocytes, might be inherently amenable to shRNA use, due to their self-limitation of viral dose ${ }^{17}$. Indeed, Sigma-Aldrich manufacturer protocol suggests using and $\mathrm{MOI}$ of 1 to deliver shRNA virus to cells. When we infected $B E(2) \mathrm{C}$ cells with LIN28B shRNA virus below an $\mathrm{MOI}$ of 1 , two out of three failed to efficiently knockdown LIN28B expression (Supp. Fig. 7 ). The challenge therefore is to titrate viral dose only as high as needed to achieve target suppression while remaining below cytotoxic levels. 
To address the shortcomings of small hairpin RNAs and to reduce potential contributions to irreproducibility in cancer research, we recommend the following practices for shRNA use. Care should be taken to titer viral supernatants, followed by use of the lowest MOI possible to effectively knockdown the gene of interest. Non-targeting scrambled sequence hairpin controls should always be used at similar $\mathrm{MOI}$ to control for the sequence-independent effects reported in this manuscript. An additional empty vector control should also be used to control for general effects of shRNA expression. Finally, phenotypes observed through effective use of shRNA knockdown should be reverted by expression of shRNA-resistant versions of the gene of interest and/or validated by either siRNA or CRISPR/Cas9 use (Supp. Fig. 6d, 8).

Reproducibility is central to scientific advancement, but has been difficult to achieve in recent years, especially in the field of cancer research ${ }^{4,18}$. Research tools such as exogenous shRNA that inappropriately effect the same cell growth inhibition that is frequently the goal of target validation studies in cancer research have almost certainly contributed, despite good faith efforts of the researchers involved. Minimizing shRNA-induced, sequence independent detrimental effects on cell growth and expression signatures will reduce false positive results and contribute to improved reproducibility in cancer and potentially other research fields.

\section{Materials and Methods:}

Cell Culture: BE(2)C (ATCC CRL-2268), HeLa (ATCC CCL-2), and K562 (ATC CCL-243) were maintained in RPMI media with $10 \%$ heat-inactivated fetal calf serum, $1 \mu \mathrm{g} \mathrm{ml}-1$ penicillin, and 1 $\mathrm{U} \mathrm{ml}-1$ streptomycin. All cell lines were purchased for the purposes of this study, are not among commonly misidentified cell lines (according to the International Cell Line Authentication Committee), and tested negative for mycoplasma contamination.

SiRNA: siRNA transfections were performed with Lipofectamine ${ }^{\mathrm{TM}}$ RNAiMAX Transfection Reagent as per the manufacturer protocol. siRNAs used: siCon/Con1 (ThermoFisher cat\# 4390843); siCon2 (ThermoFisher cat\# 4390846); si LIN28B (ThermoFisher cat\# 4392420, identifier s52477); siMYCN (ThermoFisher cat\# 4392420, identifier s9134); si $A B L 1$ (ThermoFisher cat\# 4390824, identifier s864); siRPL3 (ThermoFisher cat\# 4392420, identifier s12142); siNAT10-1 (ThermoFisher cat\# 4392420, identifier s30491); siNAT10-2 (ThermoFisher cat\# 4392420, identifier s30492).

Lentivirus: Lentiviral shRNA particles were prepared as previously described ${ }^{19,20}$. Viral titers were determined using Takara Lenti- $\mathrm{X}^{\mathrm{TM}} \mathrm{GoStix}^{\mathrm{TM}}$ as per the manufacturer protocol. ShRNA constructs used: shCon/Con1 (SHC216, sequence 5'- CGTGATCTTCACCGACAAGAT-3'); shCon2 (SHC204, sequence 5'- CGTGATCTTCACCGACAAGAT-3'); shEV (SHC201-no insert); shLIN28B1/B1 (TRCN0000144508, sequence 5'-CCTGTTTAGGAAGTGAAAGAA-3'); shLIN28B2/B2 (TRCN0000122599, sequence 5'-GCCTTGAGTCAATACGGGTAA-3'); shLIN28B3/B3 (TRCN0000122191, sequence 5'-GCAGGCATAATAAGCAAGTTA-3'); shNAT10-1 (TRCN0000296411, sequence 5'-TTGCTGTTCACCCAGATTATC-3'); shNAT10-2 (TRCN0000035702, sequence 5'-CGCAAAGTTGTGAAGCTATTT-3'). CRISPR/Cas9 constructs used: V2-Cas9 (lentiCRISPRv2, Addgene plasmid \#52961), V2-GFP1 was created by cloning eGFP sgRNA sequence 5'-GGGCGAGGAGCTGTTCACCG-3' into the lentiCRISPRv2 vector. V2GFP2 was created by cloning eGFP sgRNA sequence 5'- GAGCTGGACGGCGACGTAAA-3' into the lentiCRISPRv2 vector. LentiCRISPRv2 was a gift from F. Zhang ${ }^{19,20}$.

Western Blotting: Western blots were performed with antibodies against LIN28B (Cell Signaling 4196), MYCN (Santa Cruz Biotechnology sc-53993), NAT10 (Proteintech 13365-1-AP), $\beta$-Tubulin (Cell Signaling 2146), and $\beta$-Actin (Santa Cruz Biotechnology sc-8342). 
Raw data processing of microarray data: The Affymetrix GeneChip miRNA 3.0 arrays were processed in $\mathrm{R}$ as follows. First, we re-annotaed the probes we loaded raw .CEL files using the 'oligo' and 'affy' packages ${ }^{21,22}$. Second, we empirically defined a background level of expression as 5.41 by determining two standard deviations above the mean probe intensity of all probes mapping to Zea mays. We excluded all non-human probes and probes mapping to un-annotated genes, leaving 1,724 probes for further analysis. We further restricted downstream analysis to the 542 probes were detected (i.e. mean expression in the group > the background defined above) in at least one experimental group. We used the mirFocus database (http://mirfocus.org/index.php) to annotate miRNAs as clustered or singletons.

Reanalysis of publicly available datasets: We reanalyzed publicly available microarray datasets. The accession numbers for theses studies are GSE46708 and GSE74622. For GSE46708, we downloaded processed data from GEO. For GSE74622, we used the R packages oligo and the annotation package pd.hugene.2.0.st to process raw .CEL files. Differential expression analysis was performed using the $R$ package limma. We used the $R$ packages clusterProfiler and ReactomePA for gene ontology enrichment analysis. For GSE46708, we used genes with log 2 fold changes $>0.5$ or $<-0.5$ for gene ontology analysis. For GSE74622, we used genes with $\log 2$ fold changes $>0.25$ or $<-0.25$ for gene ontology analysis. Clustering and heat map visualization were performed the pheatmap package.

Literature analysis: We downloaded xml files from ftp://ftp.ncbi.nlm.nih.gov/pub/pmc and wrote customized scripts to parse these $\mathrm{xml}$ files and query combinations of keywords throughout each manuscript. These scripts are available from the authors upon reasonable request.

\section{References:}

1. Jackson, A. L. Widespread siRNA 'off-target' transcript silencing mediated by seed region sequence complementarity. RNA 12, 1179-1187 (2006).

2. Jackson, A. L. et al. Expression profiling reveals off-target gene regulation by RNAi. Nat. Biotechnol. 21, 635-637 (2003).

3. Grimm, D. et al. Fatality in mice due to oversaturation of cellular microRNA/short hairpin RNA pathways. Nature 441, 537-541 (2006).

4. Begley, C. G. \& Ellis, L. M. Drug development: Raise standards for preclinical cancer research. Nature 483, 531-533 (2012).

5. Martin, J. N. et al. Lethal toxicity caused by expression of shRNA in the mouse striatum: Implications for therapeutic design. Gene Ther. 18, 666-673 (2011).

6. Beer, S. et al. Low-level shRNA cytotoxicity can contribute to MYC-induced hepatocellular carcinoma in adult mice. Mol. Ther. 18, 161-170 (2010).

7. Powers, J. T. et al. Multiple mechanisms disrupt the let-7 microRNA family in neuroblastoma. Nature 535, 246-251 (2016).

8. Kugel, S. et al. SIRT6 suppresses pancreatic cancer through control of Lin28b. Cell 165, 1401-1415 (2016).

9. Russo, A. et al. Human rpL3 induces $\mathrm{G}_{1} / \mathrm{S}$ arrest or apoptosis by modulating $\mathrm{p} 21$ (waf1/cip1) levels in a p53-independent manner. Cell Cycle (2013). doi:10.4161/cc.22963 
10. Jubierre, L. et al. BRG1/SMARCA4 is essential for neuroblastoma cell viability through modulation of cell death and survival pathways. Oncogene 35, 1-12 (2016).

11. Wang, L. et al. Intracellular CD24 disrupts the ARF-NPM interaction and enables mutational and viral oncogene-mediated p53 inactivation. Nat. Commun. 6, (2015).

12. Pan, Q. et al. Disturbance of the microRNA pathway by commonly used lentiviral shRNA libraries limits the application for screening host factors involved in hepatitis $\mathrm{C}$ virus infection. FEBS Lett. 585, 1025-1030 (2011).

13. Van Gestel, M. A. et al. ShRNA-induced saturation of the microRNA pathway in the rat brain. Gene Ther. 21, 205-211 (2014).

14. Mendell, J. T. miRiad Roles for the miR-17-92 Cluster in Development and Disease. Cell 133, 217-222 (2008).

15. Khuu, C., Utheim, T. P. \& Sehic, A. The Three Paralogous MicroRNA Clusters in Development and Disease, miR-17-92, miR-106a-363, and miR-106b-25. Scientifica 2016, (2016).

16. O’Donnell, K. A., Wentzel, E. A., Zeller, K. I., Dang, C. V. \& Mendell, J. T. c-Myc-regulated microRNAs modulate E2F1 expression. Nature 435, 839-843 (2005).

17. Manier, S. et al. The LIN28B/let-7 axis is a novel therapeutic pathway in multiple myeloma. Leukemia (2016). doi:10.1038/leu.2016.296

18. Ioannidis, J. P. A. Why most published research findings are false. PLoS Medicine 2, 06960701 (2005).

19. Sanjana, N. E., Shalem, O. \& Zhang, F. Improved vectors and genome-wide libraries for CRISPR screening. bioRxiv 11, 006726 (2014).

20. Shalem, O. et al. Genome-scale CRISPR-Cas9 knockout screening in human cells. Science 343, 84-7 (2014).

21. Carvalho, B. S. \& Irizarry, R. A. A framework for oligonucleotide microarray preprocessing. Bioinformatics (2010). doi:10.1093/bioinformatics/btq431

22. Gautier, L., Cope, L., Bolstad, B. M. \& Irizarry, R. A. Affy - Analysis of Affymetrix GeneChip data at the probe level. Bioinformatics (2004). doi:10.1093/bioinformatics/btg405 
bieRxiy prepript doi:https://ploi.org/10.1101/372920; this version posted December 11, 2018. The copyright holder for this preprint (which was -

Figure 1. shRNA expression impairs cell growth. (a,b) Immunoblot for LIN28B in BE(2)C neuroblastoma cells transduced with indicated lentivirus (a) or siRNA (b) for 2 days. (c,d) Cell cycle profiles of PI stained cells treated as in $a$ and $b$, respectively. (e) Growth analysis of K562 leukemia cells infected with the indicated lentivirus (MOI 20). (f) Cell growth analysis of K562 cells transfected with the indicated siRNA. (EV, empty vector; Con, non-targeting shRNA control; B1/B2/B3, LIN28B targeting shRNAs)

Figure 2. Control shRNAs inhibit cell growth. $(\mathbf{a}, \mathbf{b})$ Relative cell growth in $B E(2) C$ and HeLa cells five days after the indicated transfections or infections. Dose for each treatment is noted. Values are relative to sham transfections or infections, respectively. $\left({ }^{*} p<0.05,{ }^{* *} p<0.01,{ }^{* * *} p<0.001\right.$, unpaired ttest).

Figure 3. Effect of exogenous shRNAs on gene expression patterning. (a) Clustering of Pearson correlation coefficients between gene expression profiles of $B E(2) C$ cells infected with the indicted lentiviral constructs. (b) Volcano plots colored by differentially expressed cell cycle-related genes. Blue indicates downregulation; Red indicates upregulation. (c) Gene Ontology analysis of genes significantly downregulated in each comparison. (d) Venn diagram of downregulated gene ontology biological processes between our dataset and datasets generated by Jubierre et al. and Wang et. al. (e) Gene ontology biological processes downregulated across the studies in $d$.

Figure 4. Effect of exogenous shRNAs on microRNA expression patterning. (a) Clustering of Pearson correlation coefficients between gene expression profiles of $B E(2) C$ cells infected with the indicated shRNA constructs. (b) Venn diagram of the 50 most downregulated microRNAs in each the $\mathrm{BE}(2) \mathrm{C}$ infections from $a$. (c) List of the 25 commonly downregulated microRNAs from $b$. OncomiRs are in blue. (d) Relative microRNA expression from clustered or singleton loci in cells from a. Values are relative to EV. (e) Analysis of relative miR-17 OncomiR cluster expression in cells from $a$. Values are relative to empty vector control. (EV, empty vector; Con, non-targeting shRNA; B1/B2/B3, LIN28B targeting shRNAs)

Supplementary Figure 1. NAT10 targeting shRNAs impair cell growth. (a) Immunoblot for NAT10 in $\mathrm{BE}(2) \mathrm{C}$ cells infected or transfected with the indicated lentiviral constructs or siRNAs, respectively. (b) Cell growth analysis of $\mathrm{BE}(2) \mathrm{C}$ cells treated as in a. (EV, empty vector)

Supplementary Figure 2. CRISPR/Cas9-mediated genetic loss of LIN28B in pancreatic cancer cells. (a) Immunoblot for LIN28B in pancreatic cancer cell lines infected with indicated Cas9-gRNA lentivirus. (sgGFP, GFP-targeting gRNA control; EX2/3.1/3.2/4, LIN28B targeting gRNAs). (b) qPCR analysis of relative let-7 expression in cells from a. Fold change relative to GFP gRNA controls. (c) Cell growth analysis of cells infected as in $a$.

Supplementary Figure 3. Effect of exogenous shRNA expression on gene expression patterning (a) Clustering of Pearson correlation coefficients between gene expression profiles. (b) Volcano plots colored by differentially expressed cell cycle-related genes. Blue indicates downregulation; Red indicates upregulation. (c) Gene Ontology analysis of differentially expressed genes. Dataset analyzed was generated by Jubierre et. al., 2016.

Supplementary Figure 4. Effect of exogenous shRNA expression on gene expression patterning. (a) Clustering of Pearson correlation coefficients between gene expression profiles. (b) Volcano plots colored by differentially expressed cell cycle-related genes. Blue, red dots indicate significantly downregulated or upregulated cell cycle related genes, respectively. (c) Gene Ontology analysis of differentially expressed genes. Dataset analyzed was generated by Wang et. al., 2017.

Supplementary Figure 5. OncomiR microRNA clusters are suppressed by exogenous shRNAs (a) List of the 40 most highly expressed microRNAs in BE(2)C EV cells as determined by microRNA microarray. OncomiRs are in blue. (b,c) Analysis of relative miR-106a and miR-106b OncomiR cluster expression in cells from infected with the indicated shRNA lentivirus. Values are relative to 


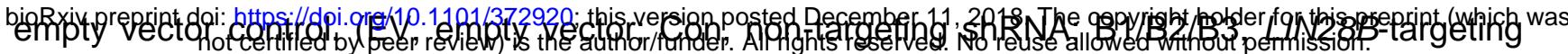
shRNAs)

Supplementary Figure 6. Literature mining of popular knockdown methods. (a) Distribution of the number of papers assembled by use of keywords "Cancer" or "Tumor" available in Nature family journal PMC entries. (b) Number of papers per journal in a containing at least one occurrence of the indicated knockdown methods. (c) Number of papers in $b$ containing at least one occurrence of indicated knockdown methods from 2007 to 2016. (d) Number of papers in $c$ containing cooccurrences of selected knockdown methods from 2007 to 2016.

Supplementary Figure 7. Immunoblot for MYCN and LIN28B in $B E(2) C$ cells infected with the indicated lentiviral constructs $(\mathrm{MOI}<1)$ for 7 days.

Supplementary Figure 8. Table of recommendations for shRNA use. 
a
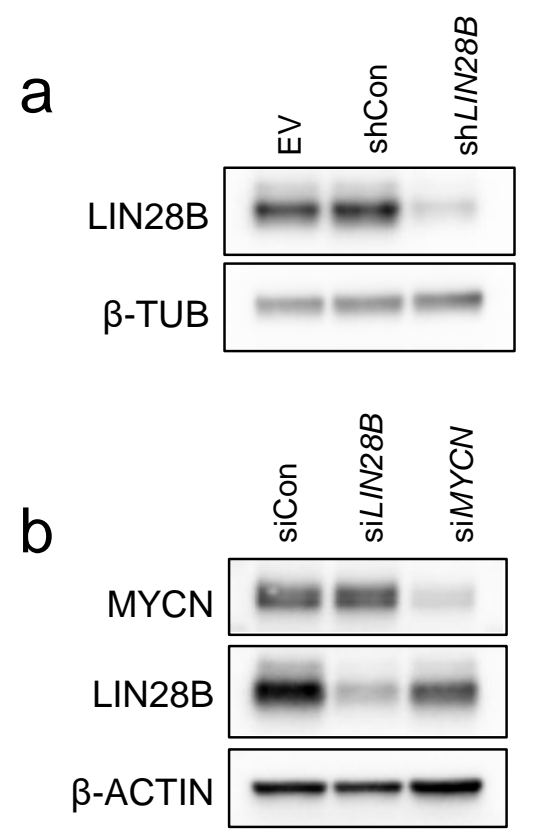

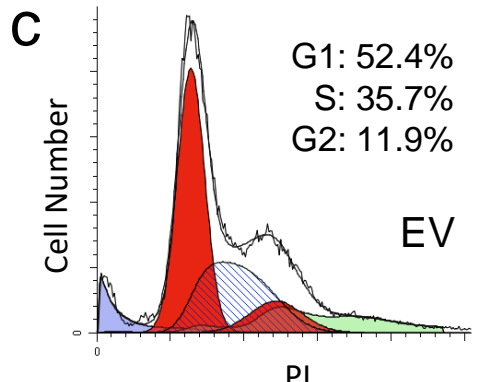

PI

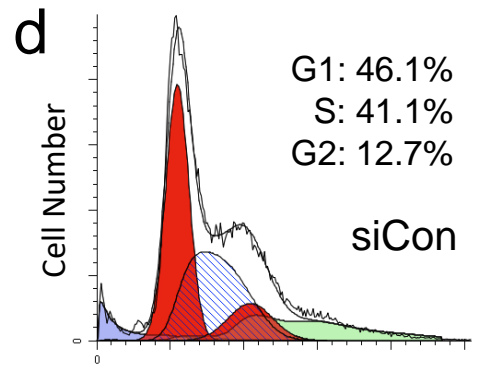

PI

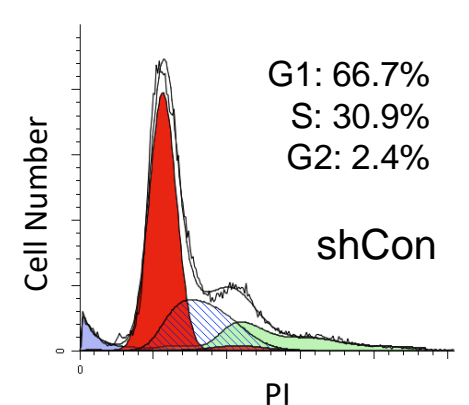

PI
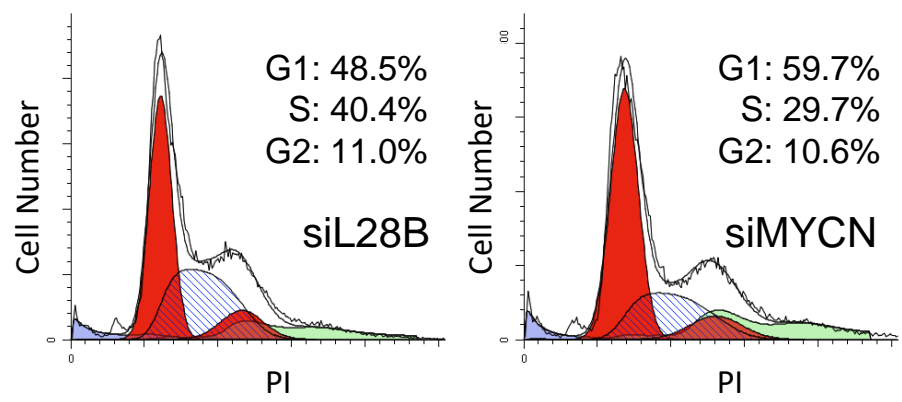

G1: $66.0 \%$

S: $25.8 \%$

G2: $8.2 \%$

shB1

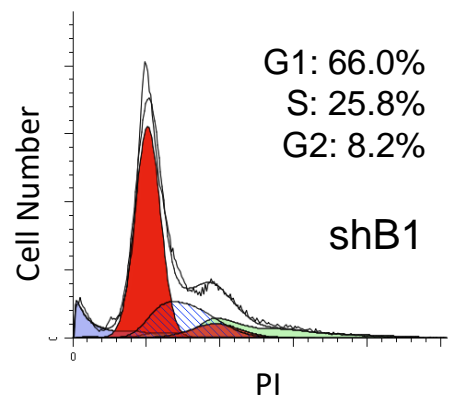

e
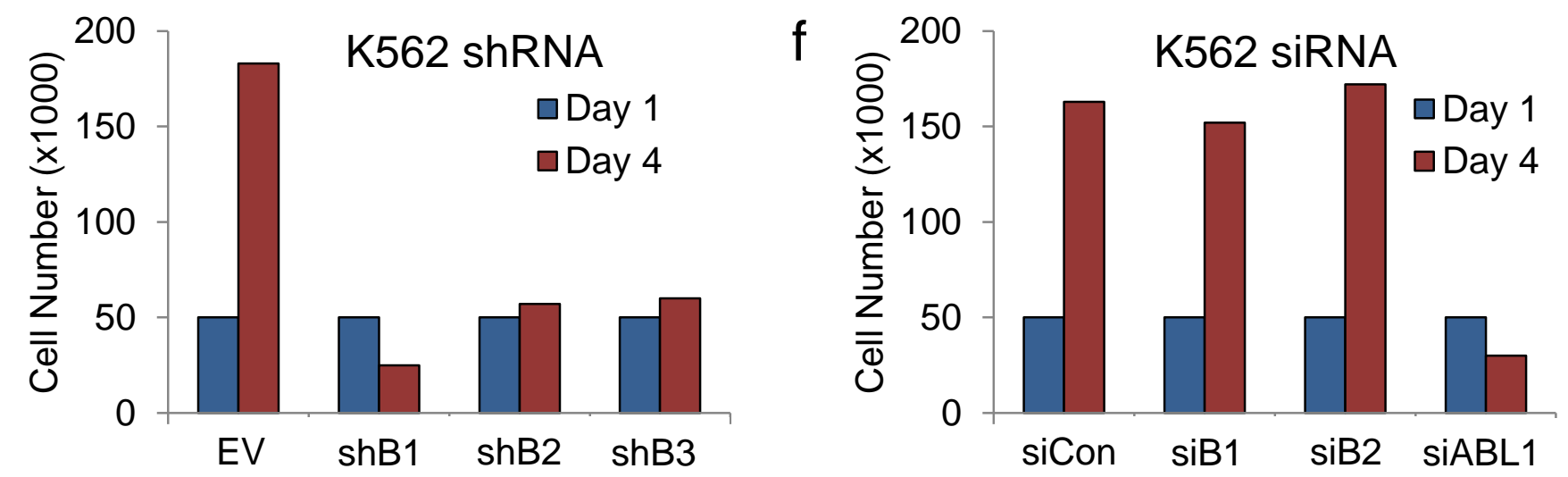


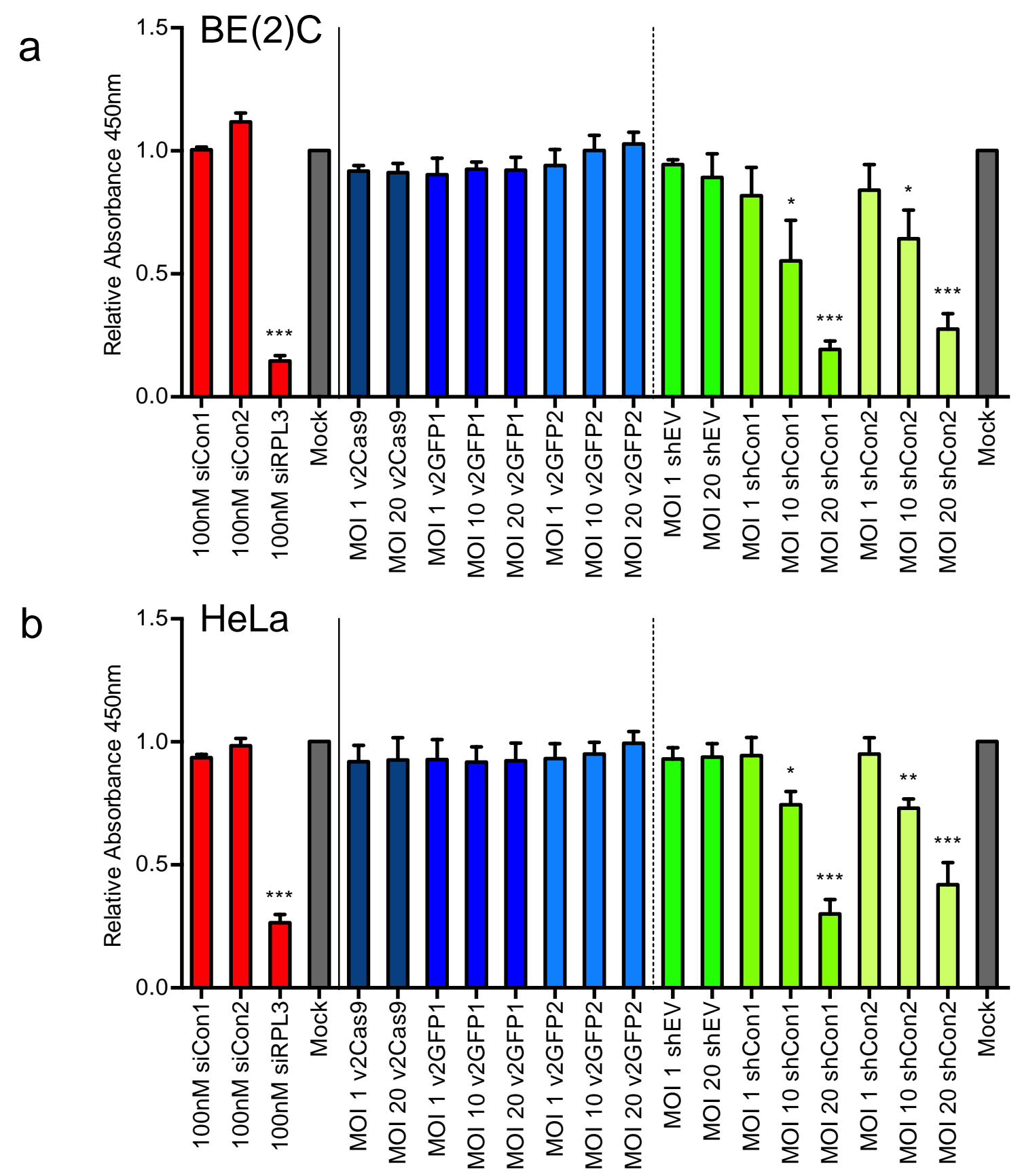


a

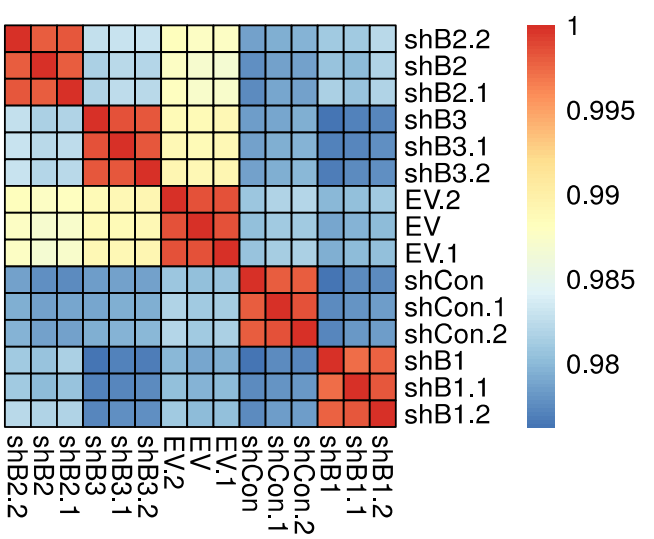

C

Downregulated
Gene Ontology Biological Processes
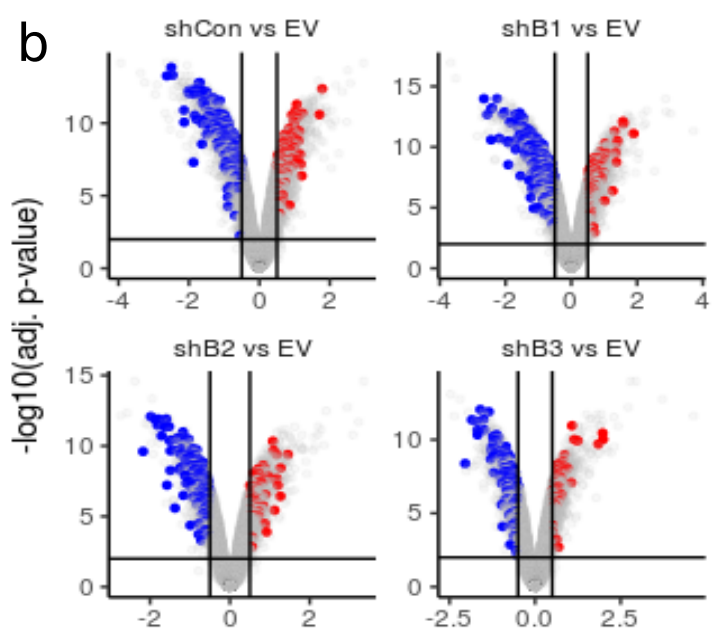

shB3 vs EV

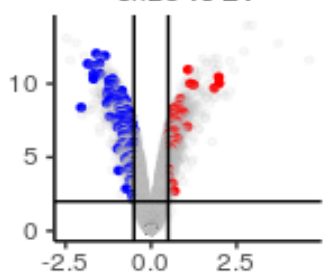

log2 fold change d

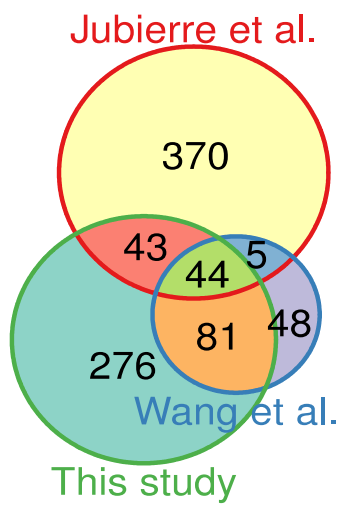

e

Shared cell cycle pathways
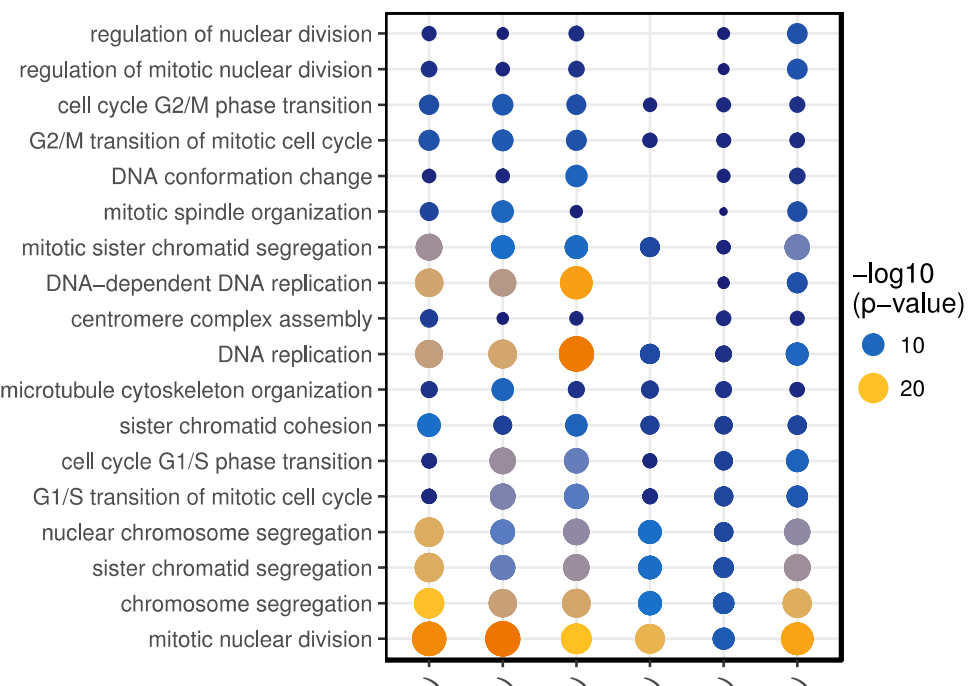

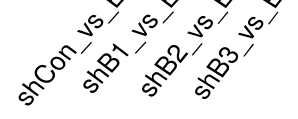

GeneRatio

mitotic nuclear division nuclear chromosome segregation sister chromatid segregation DNA-dependent DNA replication DNA replication mitotic sister chromatid segregation sister chromatid cohesion

DNA synthesis involved in DNA repair cellular amino acid metabolic process spindle organization

DNA replication initiation microtubule cytoskeleton organization cell cycle $\mathrm{G} 1 / \mathrm{S}$ phase transition

$\mathrm{G} 1 / \mathrm{S}$ transition of mitotic cell cycle

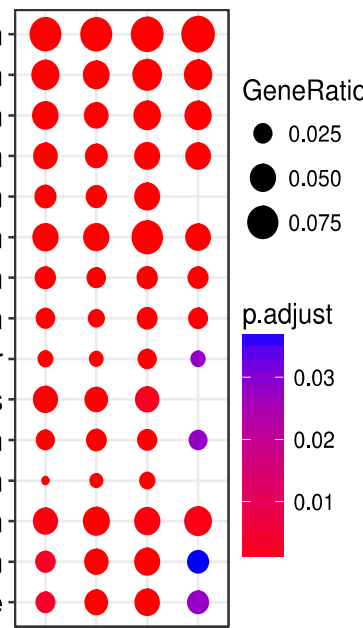
से बे बे

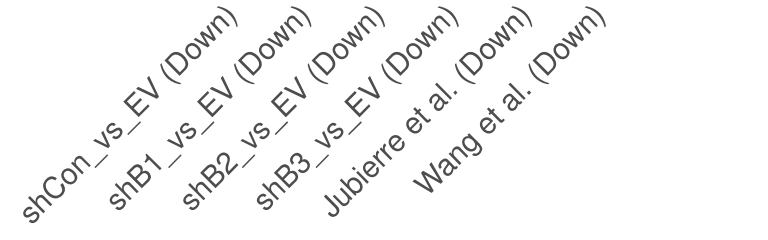



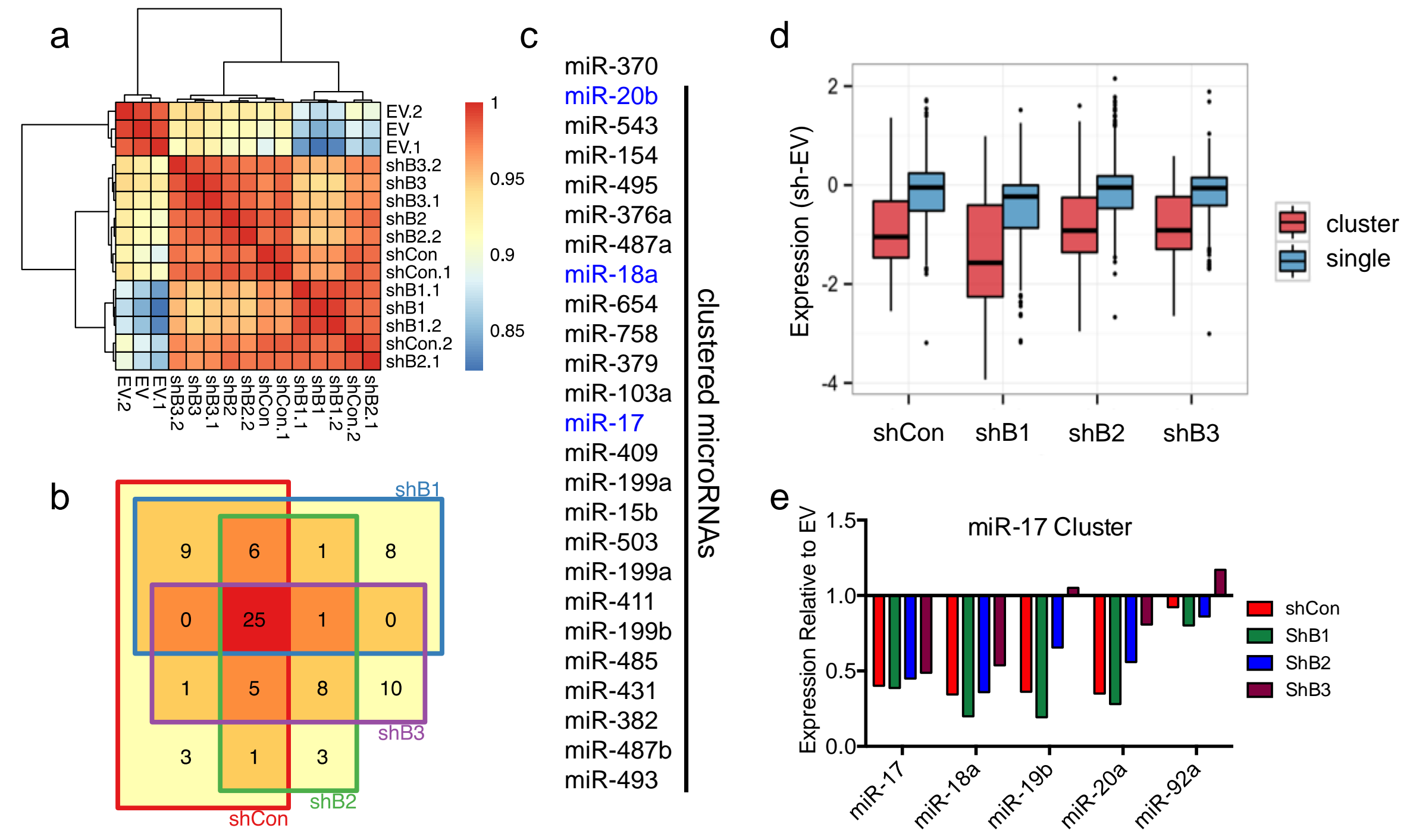


\section{Supplemental Figure 1}

a

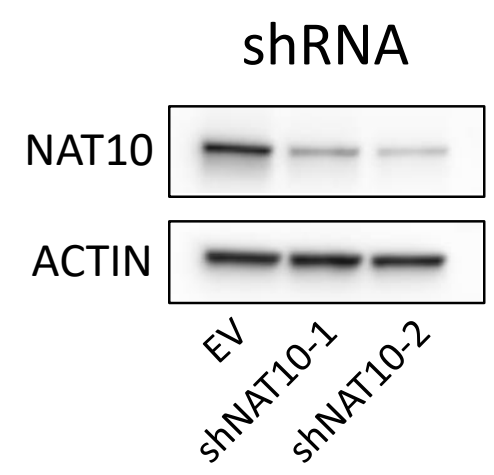

b

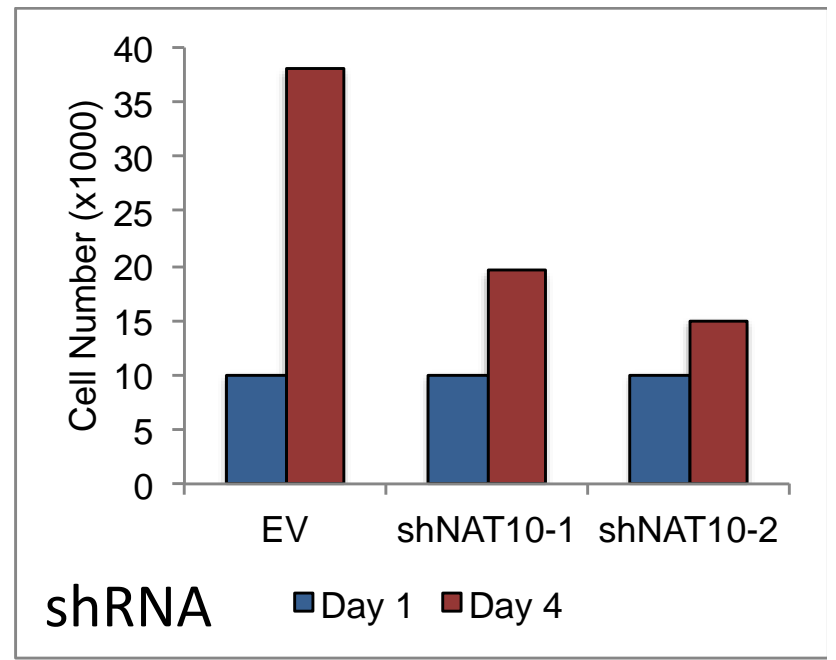

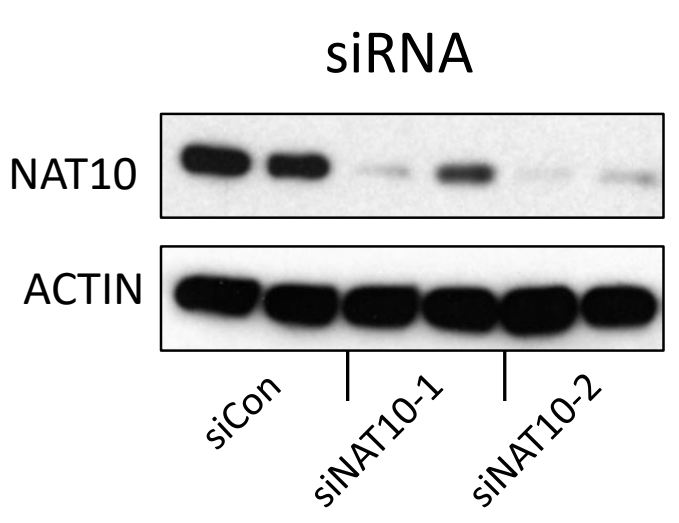

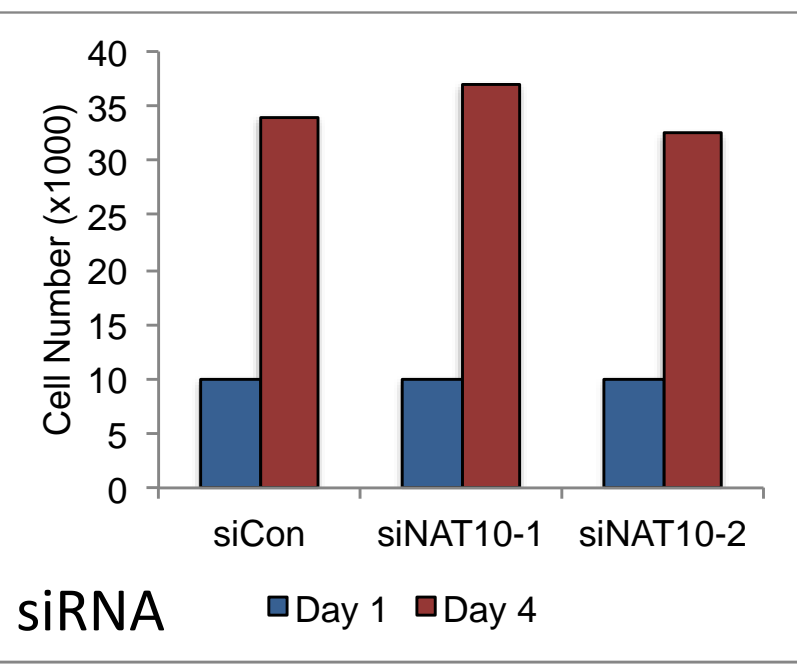




\section{Supplemental Figure 2}

a

LIN28B

Tubulin
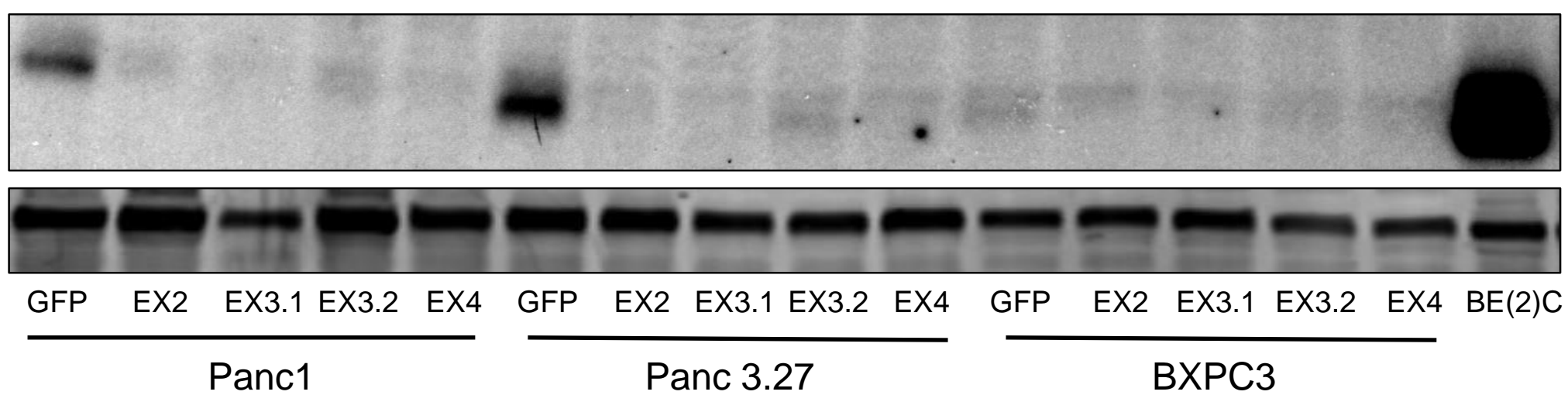

b
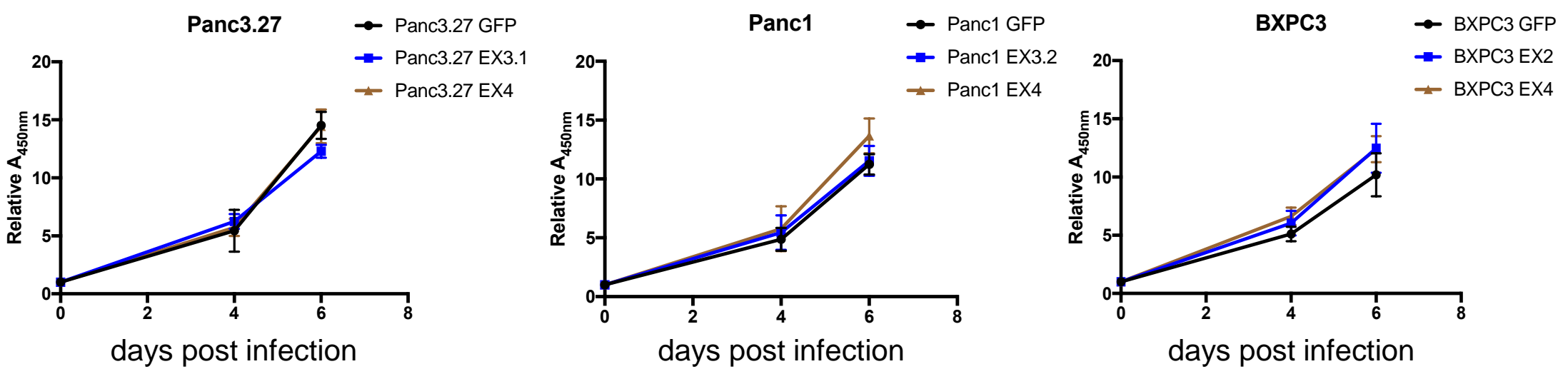


\section{Supplemental Figure 3}

a
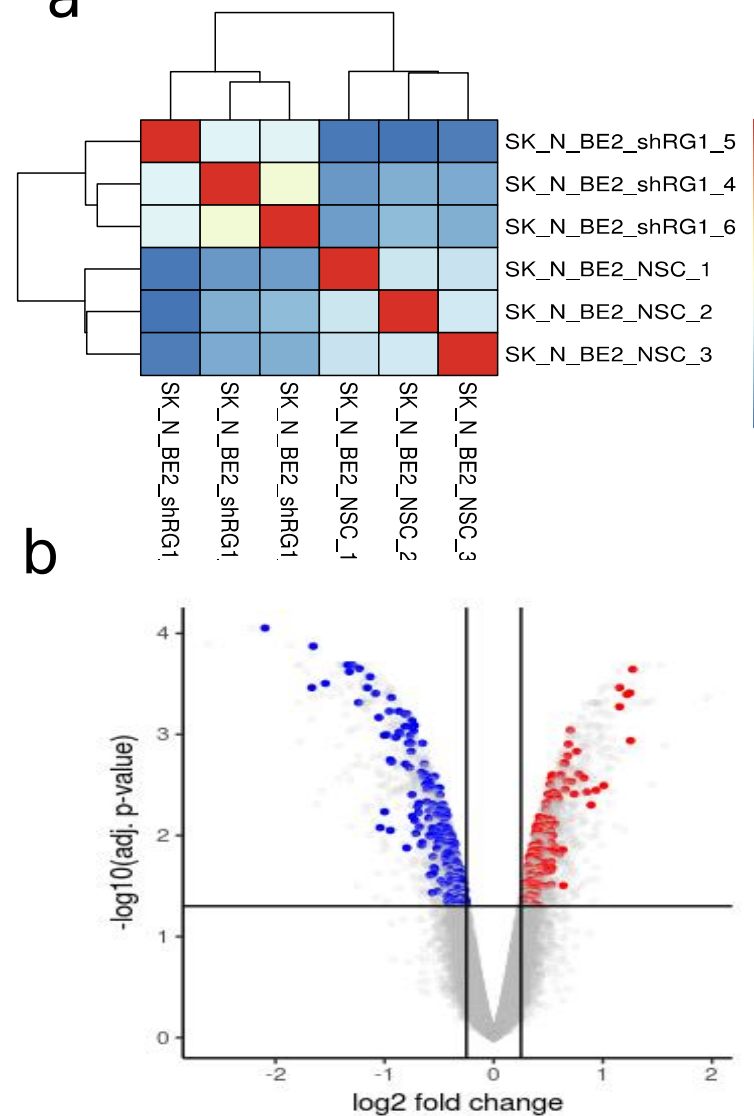

C

Gene Ontology nBiological Processes

mitochondrial RNA mRA processing

tRNA metabolic process transcription from mitochondrial promoter ncRNA processing amino acid activation methylation mitochondrial translation macroautophagy
tRNA aminoacylation cellular amino acid metabolic process tRNA aminoacylation for protein translation branched-chain amino acid catabolic process ribonucleoprotein complex biogenesis positive regulation of protein kinase activity mitotic nuclear division chromosome segregation regulation of protein serine/threonine kinase activity
sister chromatid segregation

positive regulation of organelle assembly plasma membrane organization nuclear chromosome segregation G1/S transition of mitotic cell cycle gland development

regulation of neuron projection development negative regulation of intracellular signal transduction regulation of cell junction assembly activation of protein kinase activity regulation of organelle assembly positive regulation of cell development cell cycle G1/S phase transition positive regulation of neurogenesis

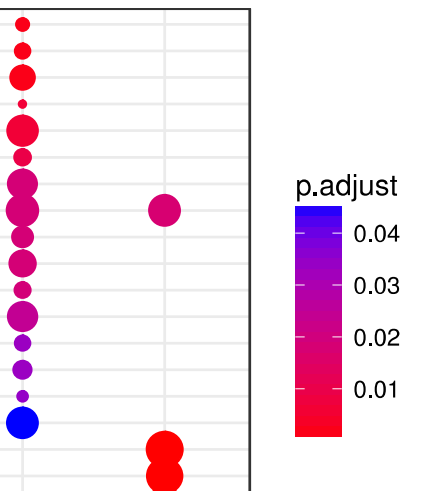

GeneRatio

- 0.01

0.02

0.03

0.04

0.05

0.06 


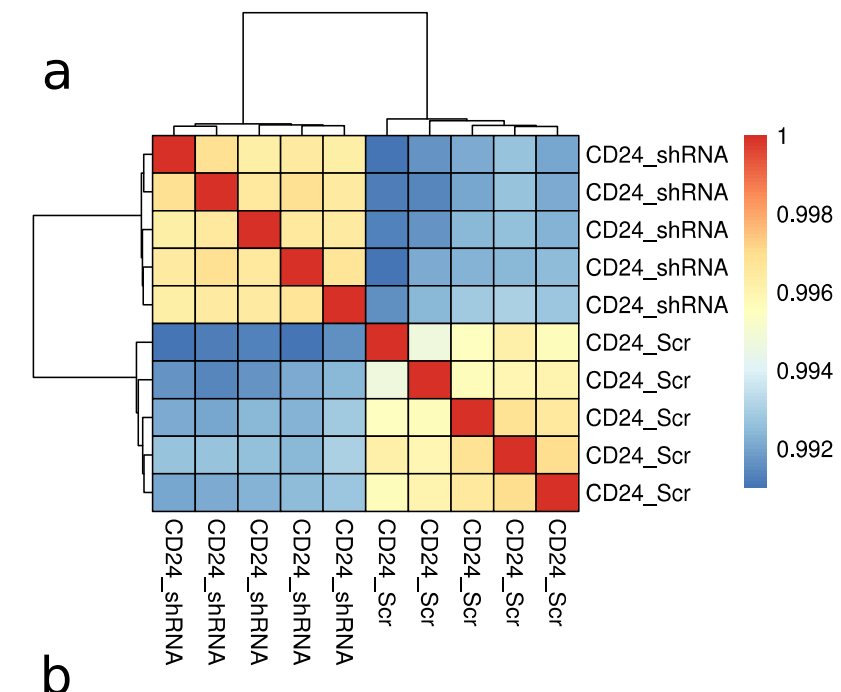

C

Gene Ontology nBiological Processes

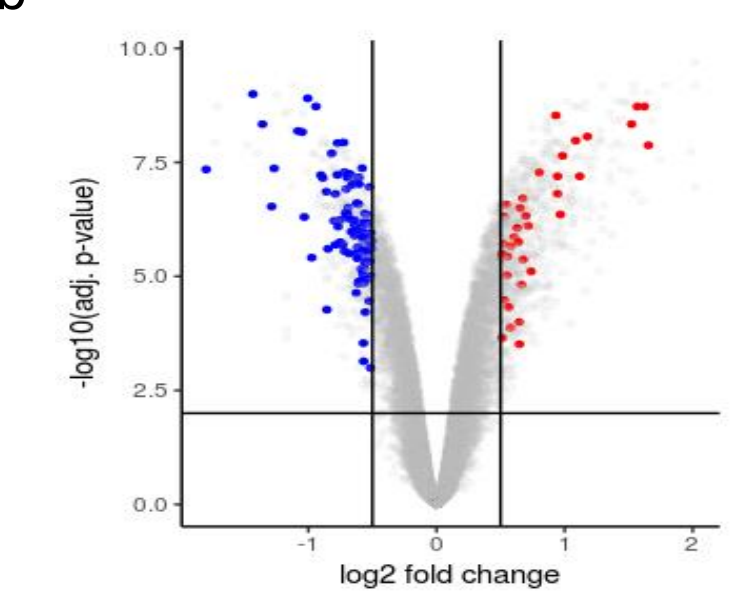

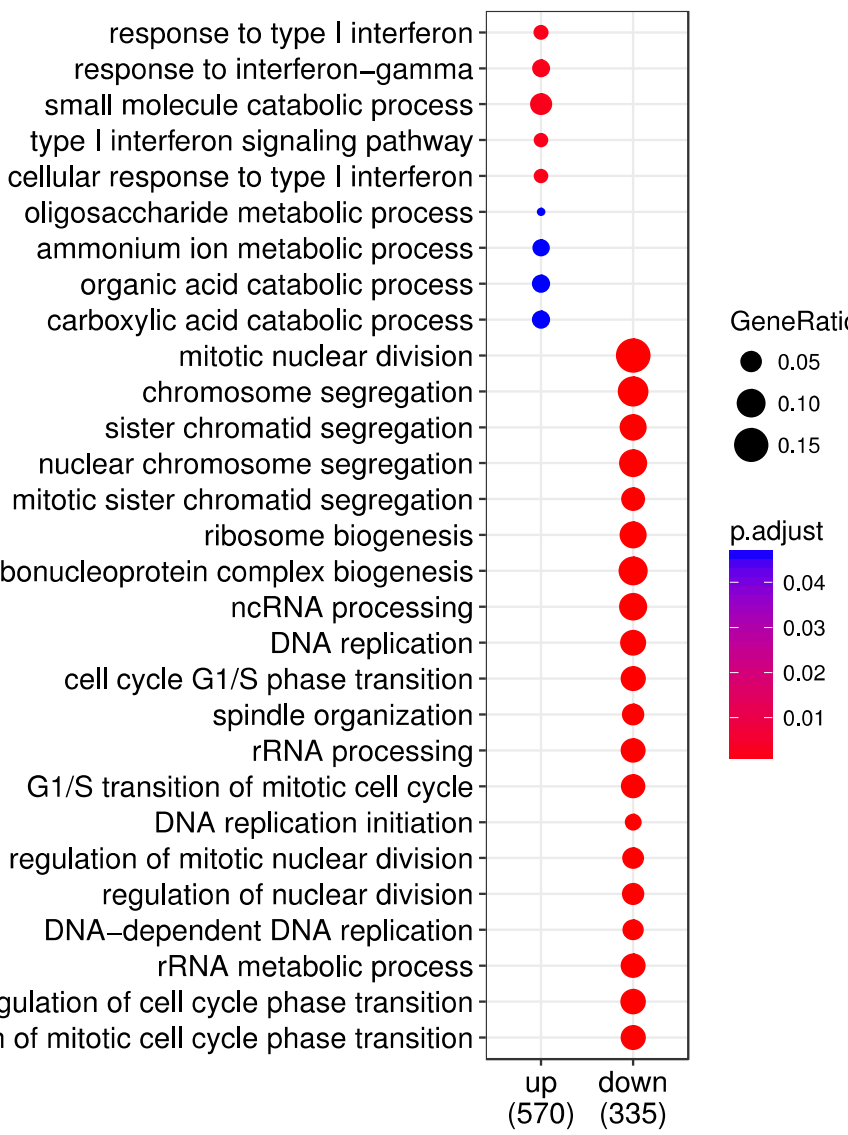

(570) (335) 


\section{Supplemental Figure 5}

a

$\begin{array}{llll}\text { miR-103a } & \text { miR-20a } & \text { miR-4454 } & \text { miR-4298 } \\ \text { miR-3960 } & \text { let-7a } & \text { miR-487b } & \text { miR-19b } \\ \text { miR-23b } & \text { miR-93 } & \text { miR-106b } & \text { miR-27b } \\ \text { miR-17 } & \text { miR-92a } & \text { miR-4787 } & \text { miR-27a } \\ \text { miR-24 } & \text { miR-320b } & \text { miR-127 } & \text { let-7e } \\ \text { miR-106a } & \text { miR-26a } & \text { miR-409 } & \text { miR-181a } \\ \text { miR-16 } & \text { miR-432 } & \text { miR-494 } & \text { miR-130b } \\ \text { miR-107 } & \text { let-7b } & \text { let-7c } & \text { miR-4466 } \\ \text { miR-3665 } & \text { miR-320c } & \text { miR-4497 } & \text { miR-379 } \\ \text { miR-23a } & \text { miR-320a } & \text { miR-18a } & \text { miR-15b }\end{array}$

b
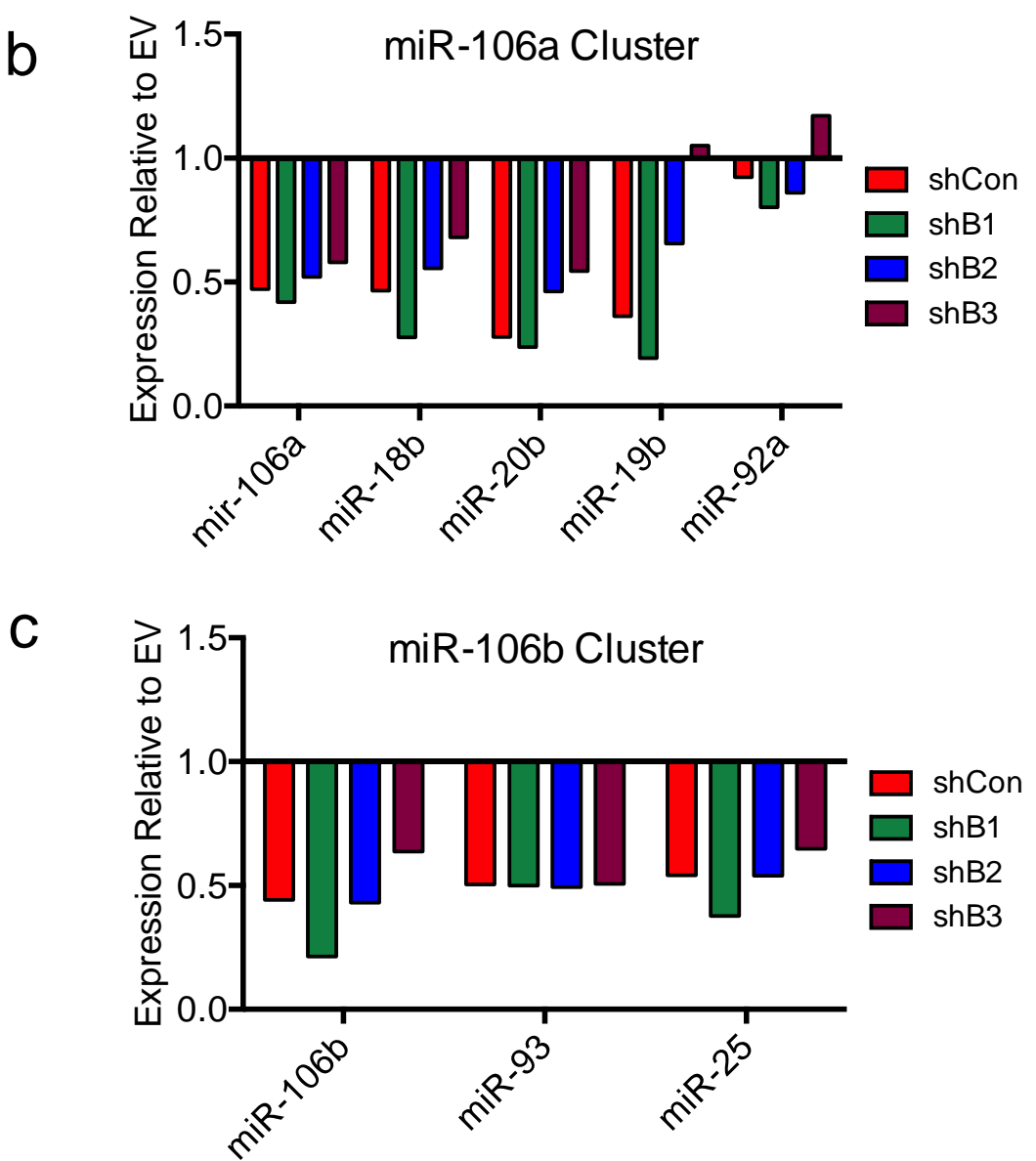


\section{Supplemental Figure 6}
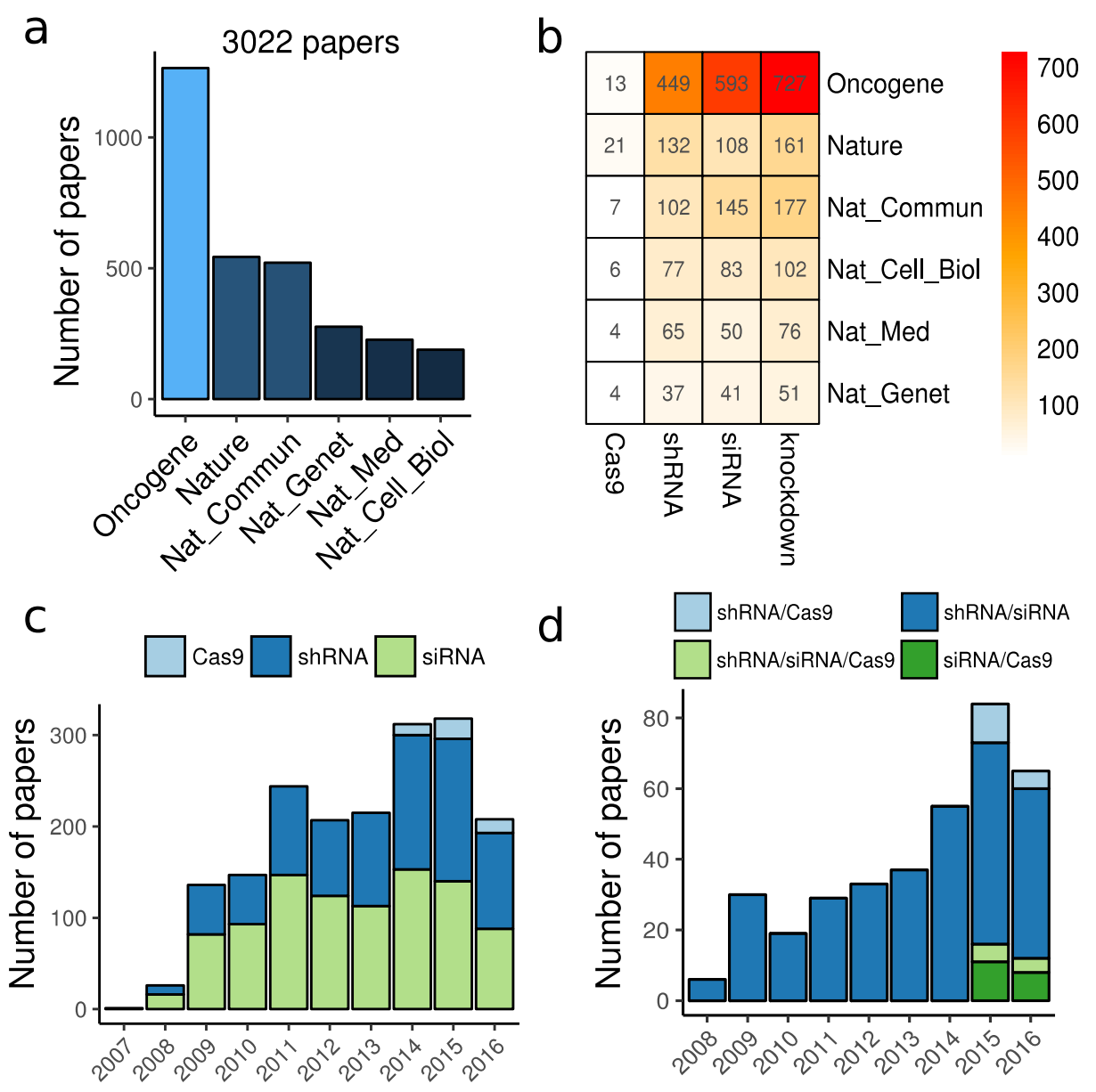


\section{Supplemental Figure 7}

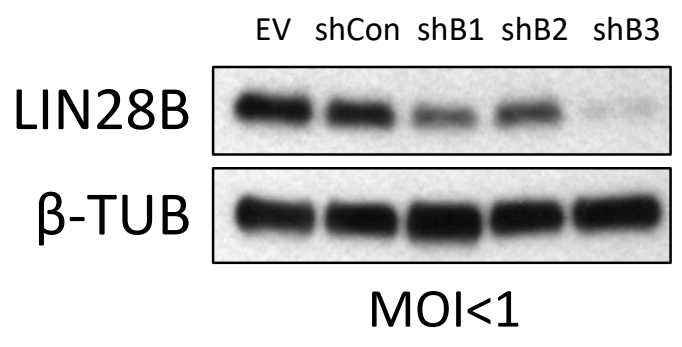




Recommendations for lentiviral shRNA use:
-Titer lentiviral supernatants. Carefully titrate
viral dose down to as low an MOI as possible
to effectively knockdown the gene-of-interest.
-Use an empty vector and at least one non-
targeting shRNA control, especially when
performing RNA-seq or other bioinformatics.
-Rescue the effect of shRNA knockdown with
gene-of-interest re-introduction.
-Validate shRNA results by siRNA knockdown
or CRISPR/Cas9 mediated knockout

